\title{
L'Année gérontologique et Les cahiers de l'année gérontologique
}

\author{
B. Vellas $\cdot$ P. Chassagne $\cdot$ C. Jeandel $\cdot$ \\ F. Nourhashémi $\cdot$ M. Verny \\ ๑) Springer-Verlag 2009
}

Créée, il y a plus de 20 ans, par les Prs J.L. Albarède, J. Pous et P. Vellas, l'Année gérontologique a évolué d'année en année. Ce fut tout d'abord un recueil annuel d'articles qui apportait aux gérontologues et aux gériatres une synthèse des avancées dans les domaines de la gérontologie sociale, clinique ou biologique. Par la suite, l'Année gérontologique a permis de publier des volumes plus spécifiques sur certains grands aspects de la gérontologie. Dans ce volume, les différents articles permettent de mieux appréhender la prise en charge spécifique des personnes âgées fragiles, et cela, dans les différents domaines de la médecine. Enfin, durant ces dix dernières années, l'Année gérontologique a régulièrement publié les actes de la SFGG. Le contenu et la diversité des communications lors de ce congrès témoignent du dynamisme, de la croissance et du développement de la gérontologie et de la médecine gériatrique en France. Du fait $\mathrm{du}$ grand nombre d'articles soumis pour publication chaque année, nous avons décidé en collaboration avec les Éditions
Springer de publier parallèlement au volume annuel de l'Année gérontologique: les Cahiers de l'année gérontologique. Chaque numéro sera coordonné par l'un des rédacteurs associés : les Prs Chassagne (Rouen), Jeandel (Montpellier), Nourhashémi (Toulouse), Vellas (Toulouse) et Verny (Paris) ; l'objectif est de prolonger l'action de l'Année gérontologique à la fois dans le domaine de la formation, mais aussi de la recherche clinique dans les thématiques importantes que sont celles spécifiques au vieillissement, notamment la nutrition, la fragilité, l'évaluation gérontologique, la cancérologie du sujet âgé, la maladie d'Alzheimer, la prévention, etc.

L'Année gérontologique et Les cahiers de l'année gérontologique restent ouverts à tous les cliniciens et chercheurs investis dans le domaine du vieillissement. Cela nous permettra de continuer à publier vos contributions, qu'il s'agisse d'articles de synthèse ou de travaux originaux, de façon encore plus rapide.

Éditorial publié dans "L'Année Gérontologique, volume 22, 2008 tome 1, pp. 9-10, (c) Serdi édition", reproduit avec l'aimable autorisation de l'éditeur.

B. Vellas · F. Nourhashémi $(\bowtie)$

Gérontopôle, CHU de Toulouse,

F-31059 Toulouse cedex 9, France

e-mail : nourhashemi.f@chu-toulouse.fr

P. Chassagne

Service de médecine interne gériatrique,

CHU de Rouen, F-38007, France

C. Jeandel

Pôle de gérontologie, centre de prévention

et de traitement des maladies du vieillissement,

CHU de Montpellier, F-75014, France

M. Verny

Centre de gériatrie du pavillon Marguerite-Bottard,

hôpital de La Pitié-Salpêtrière, F-75651 Paris, France 